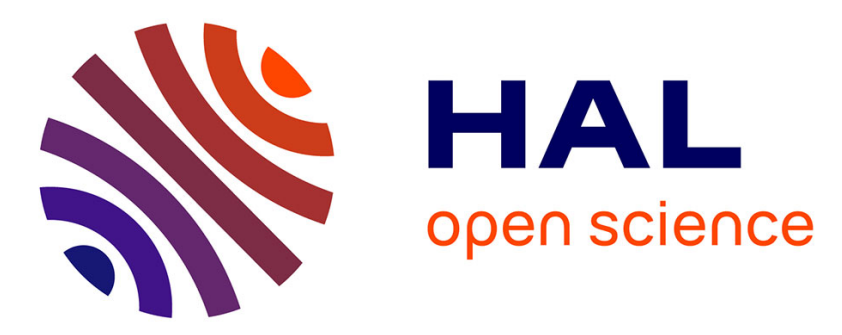

\title{
Giving up work after cancer: An exploratory qualitative study of three clinical cases
}

Géraldine de Blasi, Evelyne Bouteyre Verdier, Laetitia Rollin

\section{To cite this version:}

Géraldine de Blasi, Evelyne Bouteyre Verdier, Laetitia Rollin. Giving up work after cancer: An exploratory qualitative study of three clinical cases. WORK, 2018, 60 (1), pp.105-115. 10.3233/WOR182712 . hal-02092083

\section{HAL Id: hal-02092083 \\ https://hal-amu.archives-ouvertes.fr/hal-02092083}

Submitted on 7 Apr 2019

HAL is a multi-disciplinary open access archive for the deposit and dissemination of scientific research documents, whether they are published or not. The documents may come from teaching and research institutions in France or abroad, or from public or private research centers.
L'archive ouverte pluridisciplinaire HAL, est destinée au dépôt et à la diffusion de documents scientifiques de niveau recherche, publiés ou non, émanant des établissements d'enseignement et de recherche français ou étrangers, des laboratoires publics ou privés. 
Work $\mathrm{xx}(20 \mathrm{xx}) \mathrm{x}-\mathrm{xx}$

DOI:10.3233/WOR-182712

IOS Press

1

\title{
Giving up work after cancer: An exploratory qualitative study of three clinical cases
}

Géraldine De Blasia,b,*, Evelyne Bouteyreb and Laetitia Rollina,c

aDepartment of Occupational and Environmental Medicine (CCPPE), Rouen University Hospital, Rouen, France

bClinical Psychopathology, Language and Subjectivity Laboratory (LPCLS -EA3278), Aix-Marseille University,Aix-en-Provence, France

cBiology and Health Information Processing Team (TIBS), Computer Science, Information Processing and Systems Laboratory (LITIS - EA 4108), Rouen University Hospital, Rouen, France

\section{Sortie de l'emploi après un cancer :}

\section{Une étude exploratoire qualitative à partir de trois cas}

\section{cliniques}

\section{Sortie de l'emploi après un cancer}

\author{
De Blasi, G
}

PhD, CHU de Rouen, CCPPE, Pavillon Aubette, 1 rue de Germont, F-76031 Rouen cedex.

Université d'Aix-Marseille (AMU), LPCLS (EA 3278), 29 Avenue Robert Schuman, F-13621

Aix-en-Provence-cedex 1.

Bouteyre, $E$.

PhD, Université d'Aix-Marseille (AMU), LPCLS (EA 3278), 29 Avenue Robert Schuman,

F-13621 Aix-en-Provence-cedex 1.

Rollin, L. 
MD, PhD, CHU de Rouen, CCPPE, Pavillon Aubette, 1 rue de Germont, F-76031 Rouen cedex.

CISMeF-TIBS-LITIS EA 4108, CHU de Rouen

Adress correspondence to Géraldine De Blasi, CHU de Rouen, CCPPE, Pavillon Aubette, 1 rue Germont, F-76031 Rouen cedex.

E-mail: geraldine.de-blasi@chu-rouen.fr

\section{8 mots - 1 tableau}

\section{Résumé}

Objectif : Cette étude qualitative exploratoire, à partir de trois cas cliniques, a pour objectif de mettre en évidence des facteurs psychologiques impliqués dans le processus de décision des patients lorsqu'ils renoncent à reprendre une activité professionnelle après le diagnostic de cancer.

Méthode : Des entretiens semi-directifs d’environ 90 minutes ont été réalisé avec deux femmes et un homme. A partir d'études de cas multiples et d'une analyse qualitative des entretiens, nous avons cherché à mettre en évidence les similarités et les singularités entre les trois situations.

Résultats : Leur comparaison met en évidence des points communs comme l'abandon du projet de reprise du travail face aux difficultés anticipées, la perte du sens du travail, l'émergence d’affects réprimés ou la réévaluation de projets professionnels suite à la maladie et le bouleversement des trajectoires de vie avec des étapes qui s’en trouvent précipitées. Il en ressort des différences, comme le sentiment d'exclusion sociale, présent chez seulement deux des sujets, et une différence de vécu face à la sortie de l'emploi selon le genre.

Conclusion : Les connaissances concernant les personnes qui cessent de travailler après un cancer font largement défaut au regard de l'abondance des recherches sur les personnes qui reprennent le travail. Il est nécessaire d'approfondir les recherches sur cette question et de 
prendre en considération cette réalité clinique pour amener les professionnels de santé ou les acteurs du maintien dans l'emploi à considérer que la reprise du travail après un cancer n’est pas souhaitée ou bénéfique à tous les patients.

Mots-clés : Cancer - Décision - études de cas - étude qualitative - Sortie de l’emploi

\section{Introduction}

D’importants progrès diagnostiques et thérapeutiques ont permis d'augmenter la survie des personnes atteintes de cancer. L'augmentation de l'incidence du cancer et le recul de l'âge de la retraite conduisent de plus en plus de patients à reprendre une activité professionnelle après le diagnostic. Des revues de la littérature internationale indiquent qu'une majorité de patients y parvient. Les taux de retour l'emploi sont proches de 63 \% malgré d'importantes disparités entre les études (1,2). Cependant, le taux d'emploi des personnes ayant été atteintes d’un cancer est inférieur à celui d'une population de référence (3-5). Le cancer a un retentissement important sur les trajectoires professionnelles. Dans les cas de sortie d'emploi suite au diagnostic, la cause la plus fréquente est la perte d'emploi (6-11) mais des personnes cessent leur activité professionnelle pour partir à la retraite ou par choix personnel. Des facteurs de vulnérabilité par rapport à la perte d’emploi sont identifiés dans la littérature (comme la localisation du cancer, l’âge au diagnostic, la catégorie socioprofessionnelle, par exemple) mais nous savons peu de choses sur ce qui conduit des patients à décider de ne plus travailler après le diagnostic de cancer.

L’objectif de cet article est de décrire les motivations ou les difficultés, qu’elles soient vécues ou anticipées, des personnes qui souhaitent cesser leur activité professionnelle et d'émettre des hypothèses, à partir de trois études de cas, sur les facteurs psychologiques qui pourraient influencer la décision de ne pas reprendre le travail après le diagnostic de cancer alors que l'état de santé n’est pas un frein à cette reprise. Le choix des études de cas, approche très utilisée et 
d’une grande valeur pour les recherches en sciences de la santé, nous apparaît pertinent pour mieux appréhender les facteurs impliqués dans le processus de décision des patients.

\section{Méthode}

\subsection{Population}

Pour le recrutement des sujets, une information sur l'étude a été diffusée auprès des professionnels de santé du CHU de Rouen. Cette étude, réalisée dans le cadre d’une recherche doctorale, a été soumise à l'avis du Comité d'éthique de l'Université d'Aix-Marseille. Le consentement éclairé des sujets volontaires a été recueilli. L’anonymat et la confidentialité des données ont été assurés à chacun des sujets. Les trois sujets recrutés pour l’étude (deux femmes et un homme) travaillaient au moment du diagnostic, puis ils ont été en arrêt maladie le temps des traitements. Ils avaient terminé les traitements curatifs et étaient en rémission au moment de l'étude. Par choix personnel, ces sujets ont décidé de cesser leur activité professionnelle. Malgré les séquelles rapportées par les sujets, leur état de santé n’était pas un frein à la reprise d’une activité professionnelle.

\subsection{Recueil des données et analyse}

Des entretiens semi-directifs, d'une durée moyenne de 90 minutes, ont été enregistrés avec l'accord des sujets. Différents thèmes ont été abordés comme l’histoire de la maladie, les répercussions du cancer et des traitements sur la vie quotidienne, la satisfaction au travail avant l'arrêt maladie, les changements perçus dans la relation au travail depuis le cancer et ce qui a motivé la décision de ne pas reprendre le travail. Ces thèmes ont été retenus car ils correspondent à des difficultés recensées dans la littérature sur le retour à l'emploi après un cancer. 
A partir d'études de cas multiples et d'une analyse qualitative des entretiens, nous avons cherché à mettre en évidence les similarités et les singularités entre les situations des sujets. 


\section{Résultats}

Les caractéristiques sociodémographiques, professionnelles et médicales des sujets, issues des entretiens, sont présentées dans le tableau $n^{\circ} 1$.

\subsection{Cas clinique $\mathrm{N}^{\circ} 1$}

Carole était assistante maternelle. Suite à un cancer du sein, elle a été licenciée pour inaptitude médicale. «Un licenciement, c'est pour faute grave, et là, c'était pour une maladie grave. J'ai eu du mal à accepter d'être mise à l'écart ». Actuellement, elle est inscrite à Pôle Emploi. Elle hésite avant d'annoncer qu'elle ne cherche pas activement d'emploi pour partir à la retraite dans quelques mois. Carole ne se « sent plus la force de travailler ». Elle exprime son besoin actuel de se recentrer sur elle-même, avec une certaine culpabilité puisqu'elle ajoute : «c'est peutêtre égoïste ». Elle a le sentiment d’avoir beaucoup «donné » au travail, d’avoir été « usée ». Carole a décidé de ne plus travailler. Elle se représente la reprise comme une étape qui serait très difficile: « Je ne me vois pas du tout retravailler, j'ai plus du tout le rythme, je n'ai plus la force, plus la capacité... Je ne me vois plus travailler dans une entreprise, [...] j'ai perdu le sens de tout ça. Là, je travaille quand j'ai envie ». Carole ne se sent pas en capacité de travailler. Sur le plan physique, elle se sent diminuée. Cette habitude de vie n'en est plus une pour elle aujourd'hui : travailler a perdu de son sens. La maladie et le fait de devoir interrompre son activité professionnelle le temps des traitements ont révélé à Carole la difficulté de son travail, une usure professionnelle et un état dépressif. Carole n’a pas d’activité professionnelle salariée mais elle considère qu'elle travaille. Elle a investi de nouvelles activités (bricolage, petits travaux, activités sportives et associatives) qui donnent du sens à sa vie quotidienne et à son avenir. A présent, probablement pour se préserver sur le plan de la santé mentale, elle fait le choix de se recentrer sur elle-même et sur sa famille qui a souffert pendant la maladie.

\subsection{Cas clinique $\mathbf{n}^{\circ} 2$}


Françoise travaillait comme secrétaire jusqu'au diagnostic d'une leucémie. Elle est en arrêt maladie depuis presque trois ans. Françoise s'interrogeait sur son projet de vie après la maladie mais elle a décidé de ne pas reprendre son ancienne activité professionnelle même si, au début de l'entretien, elle laisse entendre qu'elle reprendra peut être un travail plus tard. Françoise n’envisage pas de retrouver son ancien poste de travail, elle anticipe de nombreuses difficultés par rapport à cette éventuelle reprise. La perspective d'un changement de poste de travail et celle d'une reprise à Temps Partiel Thérapeutique, semblent représenter des obstacles à son retour au travail. Le fait de ne plus être en capacité de retrouver son ancien poste l'a vraisemblablement affectée sur le plan narcissique et entrave l'investissement de nouvelles activités professionnelles, estimées moins valorisantes. Le manque de continuité, imposé par le temps partiel thérapeutique est perçu comme un frein à l'investissement au travail. Françoise a maintenu un lien assez soutenu avec le travail. Malgré ce maintien des liens, Françoise «pense que la reprise est très difficile, recréer des liens, retrouver les gens... Se réimprégner du monde professionnel, c'est délicat, je pense ». Elle semble se sentir en décalage par rapport à son entourage professionnel. Le cancer a précipité un projet qu'elle avait déjà avant d'être malade : « J'avais déjà dans l’idée, avant de tomber malade, de changer d'activité ». Mais la maladie a fait basculer ce projet : «La maladie a certainement précipité ce changement et le fait de vouloir arrêter de travailler ». Françoise évoque son âge, la période de vie qui est la sienne actuellement. Si le cancer était survenu à un autre moment de sa vie, la question de la reprise du travail se serait posée en d'autres termes. "Il y a l'âge aussi, j'ai 50 ans, si j'en avais 30 ou 40, je serais sûrement déjà retournée travailler, je ne serais pas restée sans activité professionnelle autant de temps, mais à 50 ans, c'est plus envisageable maintenant de m'arrêter ». La maladie bouleverse le rapport au temps, à 50 ans, il lui paraît raisonnable de s’arrêter, comme si sa vie professionnelle était terminée et qu'elle avait trouvé un nouvel 
équilibre de vie. Françoise trouve satisfaction et épanouissement dans ses activités quotidiennes sociales, domestiques ou de loisirs.

\subsection{Cas clinique $\mathrm{N}^{\circ} 3$}

Philippe était gestionnaire d'atelier. Un lymphome lui a été diagnostiqué il y a deux ans. Il est en arrêt de travail depuis. Pendant son arrêt maladie, le service dans lequel il exerçait a fermé. Les membres de son équipe ont été affectés à différents postes au sein de l'entreprise. Au cours de sa carrière professionnelle, Philippe a été exposé à l'amiante et s’est posé des questions quant à une potentielle origine professionnelle de son cancer. Il se sent en moins bonne santé qu'avant le diagnostic. Il présente des séquelles invalidantes au quotidien et qui ont des répercussions sur sa vie sociale, particulièrement sur ses sorties et ses loisirs. La maladie a bouleversé les habitudes de vie de Philippe et a stoppé ses projets. Il lui est encore difficile, même après la fin des traitements, de retrouver ses marques. La reprise du travail était un objectif pour Philippe après la fin des traitements : "Ça aurait été très important de reprendre pour moi, je l'avais prévu déjà et j’ai prévenu : "Allez, après la chimio, dans 6 mois je reprends », et puis ça ne s'est pas passé comme ça ». Pendant son arrêt maladie, son service a fermé. Deux choix s’offraient à lui, soit il reprenait une activité professionnelle dans un nouveau service, avec de nouveaux collègues, mais « dans ce contexte, la reprise aurait été difficile » soit il acceptait un départ anticipé à la retraite. Philippe a accepté ce départ avec frustration et un sentiment d’inachevé. Ces changements au sein de l'entreprise ont conditionné l'éventuelle reprise de Philippe, la situation aurait été différente si son poste de travail avait été maintenu. Il éprouve le sentiment de ne plus avoir sa place au travail. Il explique avoir un autre regard sur la vie et avoir changé sa façon d’être avec les autres depuis la maladie : « La santé est passée au premier plan, c'est le sujet numéro 1[...] J'ai de la chance que cet épisode se soit bien passé et qu'il n’y ait pas eu de suite [...] ça ne se passe pas comme ça pour tout le monde... Ça remet les 
idées en place, on change de regard sur les autres, on est plus attentif et compréhensif, ça remet les pendules à l'heure... ».

\subsection{Similarités, singularités entre les trois cas cliniques}

Nous allons comparer ces trois cas, à partir des aspects communs qu'ils partagent mais aussi de leurs singularités, afin de mettre en évidence les facteurs qui ont influencé la décision de ne pas reprendre le travail après le diagnostic de cancer.

\subsubsection{La reprise du travail anticipée comme une étape difficile}

Les sujets sont confrontés à des changements de situation professionnelle, du fait de leur état de santé (Carole est licenciée pour inaptitude médicale et Françoise ne pourra pas reprendre son ancien poste de travail) ou d'une restructuration de l'entreprise pour Philippe.

La reprise du travail, dans un contexte nouveau, apparaît comme une étape difficile. Tous évoquent des séquelles plus ou moins invalidantes au quotidien, et leurs possibles répercussions au travail. Carole et Françoise ne se sentent pas en capacité (physique ou psychologique) de reprendre une activité. Pour Carole, le travail a perdu de son sens, tandis que pour Françoise, une activité moins valorisante que celle de son ancien poste ne présente pas d'intérêt particulier. Le travail ne fait plus partie de leurs habitudes de vie.

Françoise souligne aussi la discontinuité induite par le temps partiel thérapeutique. Cette perspective semble altérer le sens et la continuité du travail. Françoise anticipe la frustration que lui causerait une reprise progressive.

"Recréer du lien" avec les autres, dans le cadre professionnel apparaît également comme une difficulté pour reprendre, même si Françoise a maintenu un contact régulier avec ses collègues. Pour Philippe, la disparition de son poste empêche toute projection dans un nouveau poste de travail. L'incertitude liée à ces changements au sein de l'entreprise, qui ont eu lieu en son absence, a fait obstacle à son projet de retour. 


\subsubsection{Travailler a perdu de son sens au regard du contexte dans lequel se trouvent les sujets}

Tous trois ont connu des situations de rupture. Ce manque de continuité dans la vie professionnelle, entre l'avant et l'après cancer, ne fait pas sens. Les activités de Carole (bricolage, petits travaux, activités sportives) sont pour elle du travail, au sens fort du terme et font partie d'un nouveau projet de vie. Avoir une activité salariée, au sein d'une entreprise, n'a aucun sens dans cette nouvelle façon de vivre.

Françoise souhaitait un changement de vie professionnelle, avant le diagnostic de cancer, elle désirait lui donner un nouveau sens mais elle n'en dit pas plus à ce sujet. Cependant, la perspective de reprendre à un poste moins gratifiant l'affecte sur le plan narcissique. Le travail est une valeur importante pour Françoise, mais en raison de ses capacités restantes après la maladie, elle préfère renoncer à sa vie professionnelle, ne se sentant pas capable de reprendre. Comme Carole, ses activités quotidiennes l'occupent beaucoup, comme un travail, et lui procure un épanouissement qu'elle estime supérieur à celui que lui octroierait une activité professionnelle.

Quant à Philippe, ce qui importait pour lui était de reprendre le travail après la fin de la chimiothérapie, pour achever ce qu'il avait commencé, même s'il avait dû participer à la fermeture de son service. La restructuration de l'entreprise, en son absence, a occasionné beaucoup de frustration. Cela a également causé une perte de repères. Il ne trouve pas de sens à travailler dans un autre service, avec de nouvelles personnes et à prendre un nouveau départ professionnel. Cette situation l'a poussé à accepter un départ à la retraite anticipé, alors qu’il aurait souhaité reprendre son activité.

\subsubsection{La maladie vient révéler des affects réprimés ou précipite la réalisation de projets}

Pour Carole, Françoise et Philippe, le diagnostic de cancer a révélé des affects ou une angoisse jusqu'alors réprimés ou a précipité la réalisation de projets. 
La maladie a révélé une souffrance au travail pour Carole. Plutôt satisfaite de son activité professionnelle, Carole a pris conscience pendant son arrêt maladie de la difficulté à exercer son métier. Elle parle "d'usure" et de son besoin actuel de se recentrer sur elle-même après "avoir beaucoup donné aux autres". La relation d'aide est au cœur de la profession de Carole, et ce type d'activité expose les professionnels concernés à un risque d'épuisement professionnel. Accueillir des enfants à son domicile l'a exposée davantage en atténuant la frontière entre vie professionnelle et vie personnelle. Actuellement, elle veut renforcer cette frontière, se recentrer sur le groupe familial et préserver sa santé psychique.

Pour Françoise, la maladie a précipité un changement qu'elle convoitait sur le plan professionnel. Cependant, ce projet a été réévalué suite à la maladie. A cette étape de sa vie, Françoise n'a plus d'ambition ou de projets professionnels, elle s'est construit une nouvelle identité et le travail n'y participe plus. Si le cancer était survenu plus tôt dans sa vie, elle n'aurait pas réagi de la même manière et elle aurait déjà repris le travail. Aujourd'hui, cela n'est plus une priorité et elle a pu faire l'expérience de nouvelles activités qui lui procurent satisfaction et participent à redéfinir une identité.

La situation de Philippe apparaît singulière et renvoie à une autre problématique de santé publique, celle des travailleurs exposés à l'amiante au cours de leur carrière professionnelle. Philippe était asymptomatique lorsqu'on lui a diagnostiqué un lymphome. Cette annonce a été particulièrement difficile. Dans ce contexte singulier, le cancer a rendu réelle la possibilité d'être malade. Philippe a élaboré une théorie étiologique pour donner du sens à son cancer (12). Il pensait trouver l'origine de son lymphome dans son histoire professionnelle. Cependant, un lien de causalité entre le lymphome et cette exposition n’a pas pu être établi par le médecin du travail. Ce cancer n’est pas la conséquence directe de son activité professionnelle. Le cancer semble avoir entraîné une augmentation de la perception du risque de cancer lié à l'amiante et de la détresse chez Philippe. 


\subsubsection{La maladie vient bouleverser une trajectoire de vie et en précipite les étapes}

Le fait d’avoir été malade du cancer vient bouleverser une trajectoire de vie, et en précipite les étapes, celle de la retraite pour Carole et Philippe, ou de l'arrêt de la vie professionnelle pour Françoise. Nous constatons une différence de vécu par rapport à cette étape de vie selon le genre et une meilleure adaptation à cette transition pour les deux femmes. Partir à la retraite (ou cesser son activité professionnelle) et avoir vécu un cancer sont deux événements de vie que les trois sujets vont vivre dans un temps relativement rapproché. Ces événements de vie vont mettre à l’épreuve leurs capacités d’adaptation et leur sentiment d’identité.

Pour Philippe, renoncer au travail semble plus difficile car il s’était projeté dans une reprise après la chimiothérapie. Cette cessation d'activité, non souhaitée et non anticipée, ne lui a pas permis de réorganiser ses investissements et a remis en cause ses projets. Nous observons une diminution de l'estime de soi, un sentiment d'inutilité et une difficulté à s’inscrire dans une nouvelle dynamique de vie. Carole et Françoise ont réorganisé leurs investissements, ce qui a rendu possible un travail de deuil de leur vie professionnelle. Les gratifications narcissiques apportées par l’investissement de leurs nouvelles activités (domestiques, sociales ou de loisirs) leur permettent de restaurer une continuité dans leur vie malgré les changements. Ces activités remplissent les fonctions du travail pour les deux femmes.

Pour Philippe, la retraite est vécue comme une rupture, une crise. Contrairement aux deux femmes, il a abandonné ses activités habituelles, de bricolage ou de loisirs, il est inactif. Il désinvestit les autres rôles sociaux qu’il endossait auparavant.

\subsubsection{L'abandon du projet de reprise du travail}

Initialement, les trois sujets avaient l'intention de reprendre une activité professionnelle. Les changements intervenus dans leurs situations, professionnelle ou personnelle, ou les difficultés anticipées par rapport à cette reprise ont conduit à un renoncement par rapport à ce projet de reprise. Cette décision, parfois vécue douloureusement, leur apporte néanmoins des bénéfices. 
Elle leur permet de rebondir, de se créer une nouvelle dynamique de vie, et c'est ce qui semble motiver le choix de renoncer à la vie professionnelle. Ces bénéfices sont de deux ordres, d'abord celui de préserver sa santé physique et/ou mentale, de prendre soin de soi (bénéfice qui est commun aux trois sujets) mais aussi d'être plus présent sur le plan familial (spécifique à Carole). Renoncer à une activité professionnelle pourrait également lui permettre de réduire l’expérience du conflit travail-famille.

\subsubsection{Un sentiment d'exclusion sociale}

Carole et Philippe n’ont pas été acteurs dans les changements qui sont intervenus dans leurs situations professionnelles respectives. Carole a été licenciée pour inaptitude médicale, ce qu’elle a vécu avec culpabilité, comme si le fait d’avoir été malade était une faute professionnelle. Objectivement, un licenciement pour inaptitude médicale intervient lorsque l'état de santé du salarié n’est plus en adéquation avec le poste de travail qu’il occupe et qu’il n’existe aucune possibilité de reclassement au sein de l'entreprise. Pour Carole, ce licenciement lui a renvoyé une incapacité à travailler en général. Physiquement et psychologiquement, elle ne sent pas capable de reprendre une activité professionnelle or c’est une femme très active. Carole ne trouve pas de sens à la vie professionnelle, elle ne sent plus en accord avec les valeurs qui animent le monde de l'entreprise. Carole s'est probablement sentie rejetée par son employeur. Depuis, elle ne semble pas pouvoir considérer qu’il existe une place pour elle dans la vie professionnelle. Elle rejette également un monde professionnel devenu étranger.

Pour Philippe, la destruction de son poste pendant son arrêt maladie a été vécu avec frustration. Philippe n’a plus de poste donc il n’a plus « sa » place au travail. Tout ce qu’il a accompli a disparu. Philippe avait annoncé à son employeur qu'il souhaitait reprendre mais le fait de ne pas se sentir attendu dans l'entreprise, que sa place n’ait pas été pensée au moment de la restructuration est vécue comme une situation de rejet. Soit Philippe acceptait une cessation 
anticipée d'activité soit il devait « refaire sa place » au travail dans des conditions plutôt adverses. 


\section{Discussion}

Nous avons pu mettre en évidence des facteurs communs qui ont conduit à la décision de ne pas reprendre d'activité professionnelle après le diagnostic de cancer chez les trois sujets :

- l'abandon du projet de reprise du travail face aux difficultés anticipées ;

- $\quad$ la perte du sens du travail ;

- l'émergence d’affects réprimés ou la réévaluation de projets suite à la maladie ;

- le bouleversement des trajectoires de vie avec des étapes de vie qui s'en trouvent précipitées.

Ces résultats apparaissent concordants avec ceux de la littérature sur le retour à l'emploi après cancer (13-15). En effet, nous retrouvons dans ces trois situations, les mêmes difficultés ou le même retentissement physique et/ou psychique que ceux vécus par les patients qui reprennent le travail. Le fait d’anticiper de façon réaliste cette reprise (avec ses bénéfices et ses difficultés), d’avoir un rapport au travail différent après la maladie semble avoir influencé la décision de ne pas reprendre d'activité professionnelle. Les bénéfices liés au fait de ne pas reprendre le travail sont estimés supérieurs à ceux liés au fait de reprendre, notamment pour préserver sa santé ou son bien être physique ou psychique.

Il faut également noter des différences entre les sujets. D’abord, le sentiment d'exclusion sociale n'est présent que chez deux des trois sujets, Carole et Philippe. Le fait d'avoir été licenciée ou d’avoir perdu son poste au cours d’une restructuration de son entreprise a été vécu comme une blessure narcissique et une situation de rejet de la part du monde du travail. Nous pouvons faire l’hypothèse que Françoise a été préservée de ce sentiment parce que son retour au travail était attendu et préparé, et que c’est elle va décliner les propositions qui lui sont faites (reprise à temps partiel thérapeutique et à un autre poste de travail). Le fait d’avoir été actrice de cette décision semble l’avoir préservée. 
Nous pouvons également observer une différence de vécu face à la sortie de l'emploi selon le genre. En effet, ce choix apparaît douloureux pour Philippe. Son activité professionnelle a largement œuvré à la construction de son identité et a servi de support narcissique. La situation semble différente pour les deux femmes qui parviennent à s'adapter à ce changement de vie. Une étude française montre qu’il existe des différences de vitesse de reprise du travail après un cancer selon le genre (16). Les hommes ont tendance à reprendre plus rapidement (dans les 6 mois suivant le diagnostic) que les femmes (dans les 18 mois qui suivent le diagnostic). Les cancers féminins n’étant pas plus invalidants que les cancers masculins, cette différence s’explique plutôt par des pressions sociales qui peuvent aussi bien être d'ordre économique qu'issues des représentations sociales du rôle sexué du chef de famille, qui travaille pour subvenir aux besoins du foyer. Philippe avait pour projet de reprendre rapidement après la fin de la chimiothérapie mais il y a renoncé face aux difficultés anticipées. Tout comme pour la reprise du travail, cesser son activité professionnelle pourrait être vécu différemment selon le genre.

Cette étude a permis de mettre en évidence des facteurs qui ont influencé la décision de ne pas reprendre une activité professionnelle après un cancer. Ces patients apparaissent comme des laissés-pour-compte, les connaissances les concernant font largement défaut au regard de l’abondance des recherches sur les personnes qui reprennent une activité professionnelle.

Il faut souligner la difficulté à recruter des personnes qui décident de ne plus travailler après le diagnostic de cancer. La tolérance sociale, face à ces sujets, semble faible. Il existe un présupposé largement admis, à savoir que la reprise du travail après un cancer a une valeur thérapeutique reconnue et qu'elle est bénéfique pour les sujets ayant souffert d’un cancer. Une pression sociale est exercée sur les patients. La société véhicule sa propre représentation de l’après-cancer : après avoir survécu à la maladie, les patients sont considérés guéris et doivent participer en travaillant, comme toute autre personne, à la vie sociale. Le travail, la productivité, 
la compétitivité, l'efficacité ou la réussite professionnelle sont des valeurs socialement prisées mais qui perdent de leur sens pour des patients qui ont vécu l’expérience du cancer. 


\section{Conclusion}

Il est nécessaire d'approfondir les recherches sur cette question et de prendre en considération cette réalité clinique pour amener les professionnels de santé ou les acteurs du maintien dans l’emploi à considérer que la reprise du travail après un cancer n’est pas souhaitée ou bénéfique à tous les patients.

Remerciements à la Ligue Nationale Contre le Cancer qui a soutenu cette recherche doctorale.

Les auteurs ne déclarent aucun conflit d’intérêt. 
References

1. Spelten, Sprangers MAG, Verbeek JHA. Factors reported to influence the return to work of cancer survivors: a literature review. PsychoOncology. 2002;11(2):124-31.

2. Mehnert A. Employment and work-related issues in cancer survivors. Crit Rev Oncol Hematol. 2011;77(2):109-30.

3. Taskila T, Martikainen R, Hietanen P, Lindbohm ML. Comparative study of work ability between cancer survivors and their referents. Eur J Cancer. 2007;43(5):914-20.

4. de Boer AGE., Taskila T, Ojajärvi A, van Dijk FJ., Verbeek JHA. Cancer survivors and unemployment. A meta-analysis and meta-regression. JAMA J Am Med Assoc. 2009;301(7):753-62.

5. Paraponaris A, Ventelou B, Malavolti L, Eichenbaum-Voline S. Le maintien dans l’activité et l'emploi. In: La vie deux ans après le diagnostic de cancer. Paris: La documentation française; 2008.

6. Short PF, Vasey JJ, Tunceli K. Employment pathways in a large cohort of adult cancer survivors. Cancer. 15 mars 2005;103(6):1292-301.

7. Choi KS, Kim E-J, Lim J-H, Kim S-G, Lim MK, Park J-G, et al. Job loss and reemployment after a cancer diagnosis in Koreans-a prospective cohort study. Psychooncology. mars 2007;16(3):205-13.

8. Mols F, Thong MSY, Vreugdenhil G, van de Poll-Franse LV. Long-term cancer survivors experience work changes after diagnosis: results of a population-based study.

Psychooncology. déc 2009;18(12):1252-60.

9. Roelen CA, Koopmans PC, Groothoff JW, van der Klink JJ, Bültmann U. Sickness absence and full return to work after cancer: 2-year follow-up of register data for different cancer sites. Psychooncology. 2011;20:1001-6.

10. Lindbohm M-L, Kuosma E, Taskila T, Hietanen P, Carlsen K, Gudbergsson S, et al. Cancer as the cause of changes in work situation (a NOCWO study). Psychooncology. 2010;20(8):805-12.

11. Institut National du Cancer. La vie deux ans après un diagnostic de cancer : de l'annonce à l’après cancer. 2014.

12. Pedinielli J-L. Les théories étiologiques des malades. Psychol Fr. 1996;41(2):137-45.

13. Main DS, Nowels CT, Cavender TA, Etschmaier M, Steiner JF. A qualitative study of work and work return in cancer survivors. Psychooncology. nov 2005;14(11):992-1004.

14. Maunsell E, Brisson C, Dubois L, Lauzier S, Frazer A. Work problems after breast cancer: an exploratory qualitative study. Psychooncology. 1999;8:467-73. 
15. Kennedy F, Haslam C, Munir F, Pryce J. Returning to work following cancer: a qualitative exploratory study into the experience of returning to work following cancer. Eur J Cancer Care (Engl). 2007;16(1):17-25.

16. Le Corroller-Soriano A-G, Malavolti L, Mermilliod C, France, Ministère du travail des relations sociales de la famille et de la solidarité, Direction de la recherche des études de l'évaluation et des statistiques. La vie deux ans après le diagnostic de cancer: une enquête en 2004 sur les conditions de vie des malades. Paris: la Documentation française; 2008. 


\begin{tabular}{|c|c|c|c|}
\hline & Carole & Françoise & Philippe \\
\hline Genre & Féminin & Féminin & Masculin \\
\hline Age & 60 & 51 & 57 \\
\hline Niveau d'études & $<$ Baccalauréat & Baccalauréat & Baccalauréat \\
\hline $\begin{array}{c}\text { Catégorie } \\
\text { Socioprofessionnelle }\end{array}$ & Employée & Employée & $\begin{array}{c}\text { Profession } \\
\text { intermédiaire }\end{array}$ \\
\hline Statut familial & Mariée & Mariée & Marié \\
\hline Nombre d'enfants & 3 & 2 & 2 \\
\hline $\begin{array}{c}\text { Localisation du } \\
\text { cancer }\end{array}$ & Sein & Leucémie & Lymphome \\
\hline Traitements & $\begin{array}{l}\text { Chirurgie } \\
\text { Chimiothérapie } \\
\text { Radiothérapie } \\
\text { Hormonothérapie }\end{array}$ & $\begin{array}{c}\text { Chimiothérapie } \\
\text { Radiothérapie } \\
\text { Greffe de } \\
\text { moelle osseuse }\end{array}$ & Chimiothérapie \\
\hline Séquelles rapportées & $\begin{array}{l}\text { Asthénie, } \\
\text { douleurs, } \\
\text { troubles de } \\
\text { l'attention et de } \\
\text { la mémoire }\end{array}$ & $\begin{array}{c}\text { Asthénie, } \\
\text { Fatigabilité }\end{array}$ & $\begin{array}{c}\text { Asthénie } \\
\text { douleurs, } \\
\text { troubles du } \\
\text { sommeil, de } \\
\text { l'attention et de } \\
\text { la mémoire }\end{array}$ \\
\hline
\end{tabular}

Tableau $n^{\circ}$ 1. Caractéristiques sociodémographiques, professionnelles et médicales des sujets 